Elsevier Editorial System(tm) for Australasian Emergency Nursing Journal Manuscript Draft

Manuscript Number:

Title: UTILITY OF PERIPHERAL INTRAVENOUS CANNULAE INSERTED IN ONE TERTIARY REFERRAL EMERGENCY DEPARTMENT: A RANDOMISED MEDICAL RECORD AUDIT

Article Type: Original Research papers

Keywords: Emergency Department; Peripheral intravenous cannulation; Cannula; intravenous device; randomised audit

Corresponding Author: Prof. Marg Fry, PhD, RN

Corresponding Author's Institution: Faculty of Nursing

First Author: Marg Fry, PhD, RN

Order of Authors: Marg Fry, PhD, RN; Bernadine Romero, BN; Alison Berry, BN

Abstract: Introduction:

Peripheral intravenous cannulation is a common intervention performed within the Emergency

Department by medical staff to assist in the delivery of care. However, previous studies have shown Emergency Department patients that have cannula device inserted are often not used. Across Australia, it is unclear the utility and frequency of use of peripheral intravenous cannulae within the emergency setting.

Method:

This is a random retrospective cohort study. Clinical information was retrieved from the Emergency Department Database, FirstNet and the paper medical record. Data from FirstNet included: patient demographic (age, gender) and clinical information (time of arrival, triage category, presenting problem, discharge diagnostic code, and disposition). Data from the patient medical record included: demographic (age, gender) and clinical information (Cannula usage and time of fluids, pharmacological agents, complications, pathology radiological investigations, other diagnostic uses).

Results:

Of the 357 randomised patients, 189 (52.9\%) had 209 peripheral intravenous cannula inserted. Of these cannulae 186 (88.9\%) were used within 72 hours. The majority of cannulae $(70.4 \% ; n=131)$ had more than one medical intervention.

Conclusion:

The majority of peripheral intravenous cannulae inserted during the Emergency Department visit were subsequently used for medical treatment. Further, the majority of devices were used for intravenous fluids and or medications and accessed for multiple interventions. For future audit purposes improved documentation of this procedure is needed.

Suggested Reviewers: Kate Curtis PhD

Trauma CNC, Trauma Service, St George Hospital

kate.curtis@sesiahs.health.nsw.gov.au

Expert in the field

Matthew Luttze 
Nurse Practitioner, St George Hospital, St George Hospital

Matthew.lutze@sesiahs.health.nsw.gov.au

Expert in the field

Opposed Reviewers: 
Dear Editor-in-Chief,

Thank you for the opportunity to submit an article to your journal, the AENJ. I would like to submit an article, 'Utility of peripheral intravenous cannulae inserted in one tertiary referral emergency department: a randomised medical record audit ' , as an original research article.

I believe this article will be of interest to your readership, as it investigates the role of cannulation within ED. All authors have approved the final manuscript, and agree with its submission. We confirm that this manuscript has not been published elsewhere and is not under consideration by another journal. All authors are listed, each author has substantially contributed to the manuscript, and no authors have a conflict of interest.

I look forward to hearing the outcome of this paper. If you have any questions, or would like any further information, please find my contact details below.

Sincerely,

Professor Margaret Fry

Director of Research and Practice Development,

Nursing and Midwifery Directorate, Northern Sydney Local Health District

Royal North Shore Hospital,

St Leonards NSW 2065

Australia

Telephone: (02) 99264693 or 0417985214

Email: margaret.fry@sydney.eu.au 


\section{UTILITY OF PERIPHERAL INTRAVENOUS CANNULAE INSERTED IN ONE TERTIARY REFERRAL EMERGENCY DEPARTMENT: A RANDOMISED MEDICAL RECORD} AUDIT

Short title: INSERTION AND USE OF PIVC IN ED

Authors

Professor Margaret Fry NP PhD (Corresponding Author)

Director of Research and Practice Development, Northern Sydney Local Health District

Faculty, University of Technology Sydney

margaret.fry@uts.edu.au

Ph:+61(2) 99264693

Address: Research and Practice Development, Nursing and Midwifery Directorate Northern Sydney Local Health District Level 7 Kolling Building, Royal North Shore Hospital St Leonards NSW 2065

Bernadine Romero,

CNC Emergency Department, St George Hospital, South Eastern Sydney Local Health District

Ph: (02) 91131655 Email: bernadine.romero@ @esiahs.health.nsw.gov.au

\section{Alison Berry}

Clinical Nurse Specialist Emergency Department, St George Hospital, South Eastern Sydney Local Health District Ph: (02) 91131680

Email: alison.berry@sesiahs.health.nsw.gov.au 


\section{CONFLICT OF INTEREST}

All authors contributed to data collection, analysis and development of manuscript. The lead author MF had no role in the review of this article by the journal.

\section{ACKNOWLEDGEMENTS}

N/A 


\section{Funding}

There was no funding provided for this study. 


\section{UTILITY OF PERIPHERAL INTRAVENOUS CANNULAE INSERTED IN ONE TERTIARY REFERRAL EMERGENCY DEPARTMENT: A RANDOMISED MEDICAL RECORD} AUDIT

\section{INTRODUCTION}

Over a billion peripheral intravenous cannulae (PIVCs) are inserted worldwide each year for health care purposes. ${ }^{1}$ Peripheral intravenous cannulation is a common intervention performed within the Emergency Department (ED). The purpose of inserting a PIVC is to obtain intravenous access to provide treatments for patients, such as those requiring the administration of pharmacological agents, intravenous fluids or pathology blood tests. Commonly across Australian EDs PIVCs are often inserted during the initial nursing assessment for the purposes of clinical pathways and or the collection of pathology investigations as a preventative measure to avoid a secondary needle puncture. However, it is unclear whether the insertion of a cannula is subsequently used for additional medical interventions.

The insertion of PIVCs has significant human resource, patient and cost implications for any health service. This is particularly so if the procedure is unnecessary as staff time is wasted and the cost of equipment over a 12 month period can be substantial. ${ }^{2}$ Therefore, the aims of the study were to explore the i) prevalence of PIVC insertion in adult patients presenting to one ED; ii) trend and patterns of usage of PIVCs; and iii) utility and or issues with PIVCs usage in the ED.

\section{BACKGROUND}

PIVC insertion is a routine ED intervention carried out by senior nursing and medical staff to help facilitate the care and treatment of patients. Across Australia, ED nurses initiate and undertake many extended practices, including PIVC insertion ${ }^{3-5}$ These extended activities have been in response to increased workload and overcrowding. ${ }^{6,7}$ 
Often in the ED PIVCs are inserted concurrently with obtaining pathology blood samples as they practice a patient will usually have their blood withdrawn using a syringe and or vacutainer needle. However, in the ED inserting a PIVC is viewed as avoiding a second needle puncture for the patient should they subsequently require intravenous treatments. ${ }^{8-10}$

A recent Australian ED study identified that fifty percent of intravenous cannulae inserted were unused or unwarranted. ${ }^{11}$ Similarly, a study conducted by Henderson et al ${ }^{9}$ identified that when PIVC access was initiated in the ED, only $42.2 \%$ (ambulance patients) and $48 \%$ (self-presenting patients) respectively had their PIVCs used. They also identified that $71.3 \%$ of the unused PIVCs in the ED were used only for pathology specimen acquisition.

The insertion of PIVCs has been associated with patient complications such as pain, discomfort, thrombophlebitis, infections and potentially sepsis., ${ }^{2,12,13}$ One of the more common organisms associated with PIVC sepsis is Straphylococcusaureusbactaeremia (SAB). SAB is commonly a hospital-acquired infection that is often associated with indwelling medical devices such as PIVCs. PIVCs can lead to the development of a SAB infection and it is estimated to occur in $0.1 \%$ of lines. ${ }^{14}$ Although this poses a relatively low risk, approximately $80 \%$ of hospitalised patients have a PIVC inserted at some point during their acute stay. ${ }^{14}$ Stuart et al conducted the study in two tertiary health services over 5 years and identified that there was a high incidence of SAB episodes associated with PIVCs inserted in emergency locations. Stuart et al goes on to note that SAB associated PIVC infections were associated with a mortality rate of $26.5 \%$. Consequently, PIVC associated SAB infections pose a significant financial cost to the healthcare system due to increased length of stay, additional antimicrobial treatment and resources required to treat the infection. According to Stuart et al, the additional costs may be as high as $\$ 20,000$ per patient. These costs and adverse health outcomes may be preventable if the PIVC was avoidable. 
Exploring the ED prevalence and utility of PIVCs could identify areas for service improvement. ${ }^{6,7,10}$ costs and staff time while optimising patient outcomes and treatment. By exploring the insertion and usage of PIVCs, we may be able to ensure the appropriate utility of the clinical procedure within the ED context.

\section{METHODS}

The study was a retrospective randomised medical record audit to identify the prevalence, insertion and utility of PIVCs within the ED setting.

\section{Site}

The study was conducted in one mixed metropolitan tertiary referral ED.

\section{Medical Record Audit}

A one month retrospective randomised medical audit of adult patients ( $>16$ years) who presented to one tertiary referral ED was conducted. A random sample of ED patient presentations during the month of July were selected for a medical record audit. The audit was to identify the prevalence of ED patients having a PIVC inserted; and whether this device was subsequently used within 72 hours of insertion. Data collected included: patient demographic (age, gender) and clinical information (type of device, designation of clinician inserting PIVC, reason for PIVC insertion, PIVC usage, and disposition).

Clinical information was retrieved from the ED Database FirstNet ${ }^{\mathrm{TM}}$ and the patients' paper medical record. Data from FirstNet included: patient demographic (age, gender) and clinical information (time of arrival, triage category, presenting problem, discharge diagnostic code, and disposition). Data from the patient medical record included: demographic (age, gender) and clinical information (PIVC usage and time of fluids, pharmacological agents, complications, pathology radiological investigations, other diagnostic uses). 
To obtain a representative randomised sample of ED patients the following sampling method was applied in consultation with a statistician. Estimated proportion of ED PIVCs was 0.4, CI 0.95, desired precision 0.05 determining a sample of 341 was required. Given missing data and availability of medical records 345 records were requested. The sample size provided a representative sample of patients having PIVC inserted.

\section{Data Analysis}

The audit examined the number of patients presenting to the ED who had a PIVC inserted. Data was analysed using IBM SPSS $\mathrm{v} 21^{\mathrm{TM}}$. Univariate analysis for the effect of each measured variable were assessed using a Chi-squared test. Age was considered as an ordered categorical variable. Chisquared analysis and a multivariate logistic regression models were applied to determine significant relationships.

\section{Ethical considerations}

This paper reports the findings of a study that adhered to the national statement on the conduct of human research by the Australian National Health and Medical Research and was approved by the Human Ethics Committees South East Sydney Local Health District Human Ethics Committee (13/201LNR/13/POWH/447). All participant data were immediately de-identified for the study and stored in a password protected Excel file accessible only to the investigators.

\section{RESULTS}

The total number of ED patient presentations for July 2013 was 5,688. A random sample was selected of 357 patients. Of the sample $208(58.2 \%)$ were female with a mean age of 52.97 years old (SD 23.0). The majority of patients arrived by private car $(n=224: 62.7 \%)$ and were allocated triage category $3(n=142: 39.8 \%)$ or triage category $4(n=117: 32.8 \%)$ (Table 1$)$.

During the study period 189 (52.9\%) patients had 209 PIVCs inserted. PIVCs were inserted by emergency staff $(n=181: 86.6 \%)$, ambulance officers $(n=28: 13.3 \%)$ or unknown operator $(n=10$ : 
4.7\%). Of the PIVCs inserted the majority ( $\mathrm{n}=186: 88.99 \%$ ) were used for treatment in either the ED or ward.

For the patients that had a PIVC the diagnoses included: musculoskeletal ( $\mathrm{n}=73: 34.9 \%)$; abdominal $(n=34: 16.2 \%)$; neurological $(n=21: 10.0 \%)$; respiratory $(n=22: 10.5 \%)$; other $(n=19: 9.0 \%)$; mental health $(n=10: 4.7 \%)$; cardiac ( $n=9: 4.3 \%)$; ear nose throat conditions $(n=8: 3.8 \%)$; alcohol and drugs $(n=3: 1.4 \%)$; obstetrics and gynecology $(n=3: 1.4 \%)$; missing $(n=3: 1.4 \%)$ cancer $(n=2: 0.9 \%)$; shock $(\mathrm{n}=2: 0.9 \%)$. There was no statistical difference found when comparing PIVC use and diagnosis (Pearson's $X^{2}$ test 12.504 df $12 \mathrm{p}=.406$ ).

Of the patients $(\mathrm{n}=189)$ who had a PIVC, $16(7.6 \%)$ required a second cannuale while $4(1.9 \%)$ had three PIVCs inserted. The primary PIVC size was unknown ( $n=157: 75.1 \%)$ for the majority of patients (Table 2). There was a statistical difference comparing triage category and cannula size $\mathrm{p}=.000$ (Pearson's $X^{2}$ test $41.65 \mathrm{df} 12$ ), although there was no difference by gender $\mathrm{p}=.593$ (Pearson's $X^{2}$ test 1.902 df 3 ). For the secondary PIVCs inserted only 10 (4.7\%) were documented for size and included: 20 gauge $(n=7: 25 \%) ; 18$ gauge $(n=2: 50 \%)$ and 16 gauge $(n=1: 25 \%)$.

Documented sites of PIVC insertion identified the most common was cubital fossa ( $n=78: 33.4 \%$ ) (Table 2). Of the PIVC inserted, 105 (50.2\%) had documented the operator's clinician level as intern or an RMO ( $\mathrm{n}=47: 44.7 \%)$, emergency nurse ( $\mathrm{n}=27: 25.7 \%)$ ambulance staff $(\mathrm{n}=23: 21.9 \%)$ and or registrar $(n=8: 7.6 \%)$.

Of the PIVCs inserted (n=209), 186 (88.9\%) were used. Of the PIVCs used in ED, intravenous fluids $(\mathrm{n}=128: 68.8 \%)$ and medication administration $(\mathrm{n}=119: 63.9 \%)$ were the common interventions (Table 2). PIVCs ( $n=186)$ were frequently accessed for multiple interventions. Of the PIVCs the majority were accessed for 2 or more interventions (Table 2). Of the PIVCs $(n=59)$ that had one intervention $38(64.4 \%)$ were for pathology alone. There was a statistical difference when comparing triage category and PIVC usage (Pearson's $X^{2}$ test $\mathrm{p}=.000: \mathrm{X} 232.316 \mathrm{df}$ 4). Using regression analysis (ANOVA) there was no difference for diagnostic groups $(\mathrm{p}=.677)$, clinician $(\mathrm{p}=.763)$, ambulance $(\mathrm{p}=.698)$ or PIVCs inserted in the ED $(\mathrm{p}=.235)$. 
Of the patients $(n=357)$ presenting to the ED the majority $(n=183: 57.7 \%)$ were discharged from ED with $131(36.6 \%)$ admitted to hospital (Table 2). For those with a PIVC, 92 (50.3\%) were discharged.

The PIVC costings for one month (Aus $\$ 13,869.00$ / monthly) were extrapolated across 12 months and identified that equipment expenditure (cannulae, injection ports, cannulae cap, medical swabs, tourniquet and PIVC dressings) was Aus $\$ 166,482.00$.

\section{DISCUSSION}

This study identified that the majority of people presenting to one ED had a peripheral cannula inserted. Of these patients over $85 \%$ had medical interventions administered through the PIVC. Further, the study demonstrated that the operators routinely made appropriate clinical decisions in determining the need for a peripheral cannula. This is in contrast to existing literature. ${ }^{9-11}$

Our study identified that the vast majority of PIVCs inserted were used, which stands in contrast to existing literature. Previous ED studies have identified that between 47 to $56 \%$ of PIVCs are unused. ${ }^{9-11}$ For example, in the USA Nguyen et al. ${ }^{10}$ reported $47 \%(n=12,475)$ of PIVCs inserted in two USA EDs remained unused. However, the study was undertaken in a Paediatric ED and the authors go on to state that clinicians tend to treat younger patients more conservatively. Similarly, Henderson et al ${ }^{9}$ in the USA reported that $56 \%$ of PIVCs inserted in one rural tertiary ED remained unused. However, the inclusion criteria were PIVC used within 60 minutes of arrival. The current study explored the utility of PIVCs for the duration of the ED stay and up to 72 hours.

One Australian tertiary referral ED study conducted by Limm et al ${ }^{15}$ reported that $50 \%$ of PIVCs inserted remained unused. While this study's demographics and characteristics were similar to the current study the findings of PIVC utility were very different with a greater percentage of cannuale used for medical interventions.

Increasing demands on ED services has resulted in the implementation of extended practice roles for ED nurses such as the introduction of the Clinical Initiatives Nurse (CIN) in New South Wales. The 
CIN role was designed to address the extended waiting times for ED patients by enabling the nurse to initiation of a PIVC and pathology investigations were key interventions that the CIN was able to initiate. However, the documentation of the PIVC operator in this study was poor. This requires further investigation and education if operator documentation is to improve. In the future, the ability to document the PIVC insertion in electronic medical records may improve the quality of audit data.

While obtaining pathology investigations from a PIVC is common ED practice there may have been instances where a venipuncture would have been sufficient. The findings of this study are equivocal as to whether the additional PIVC usage was done because of convenience. However, the variety and frequency of PIVC interventions would suggest that for the majority of patients they were spared an additional clinical procedure given the multiple interventions per patient.

The insertion of unnecessary PIVCs in the ED could lead to negative patient outcomes, and unnecessary utilisation of staff time. Unnecessary and unused PIVCs can also contribute to additional financial costs if deemed inappropriate or unnecessary. The PIVC costing audit identified that the procedure has substantial costs for EDs over a 12 month period. Therefore, decreasing the amount of unnecessary PIVCs may significantly reduce hospital costs. Further investigation is required of the cohort who had a cannula inserted, only had pathology investigations undertaken and were subsequently discharged home. Further investigation is needed to determine whether more appropriate clinical pathways and or education is needed to reduce unnecessary ED PIVCs particularly for this group.

\section{Limitations}

This retrospective study was conducted in one Australian metropolitan ED and therefore the results may not be representative of other EDs. While the study site was a mixed ED only adult PIVCs were reviewed and so the findings are unlikely to apply to the paediatric population. Data was only collected over a one month period and as a result there may be sampling bias and consequently this may not be representative of the prevalence and use of ED PIVCs. 
This study did not aim to compare the differences between used and unused cannuale for presenting problem. Prospective studies need to be conducted to determine whether a cannula was inappropriately or unnecessarily inserted for patient care. Further studies need to explore the use of ED cannulae subsequently used in ward areas.

\section{CONCLUSION}

The ED study demonstrated that the majority of PIVCs were used for multiple medical interventions. It remains unclear whether interventions may have been initiated for convenience given that a PIVC had already been inserted. Further prospective studies need to be conducted to explore the use of PIVCs for convenience rather than necessity particularly for discharged patients.

The study highlighted the need for improved PIVC documentation and in particular for the number of PIVC attempts, insertion site, cannulae size and operator. The development and use of electronic medical records may improve compliance of documentation and more readily enable quality auditing of this clinical procedure give cost, patient outcomes and time implications. 


\section{REFERENCES}

1. Alexandrou E, Ray-Barruel G, Carr PJ, et al.: International prevalence of the use of peripheral intravenous catheters. J Hosp Med 2015.

2. Gonzalez Lopez JL, Arribi Vilela A, Fernandez del Palacio E, Olivares Corral J, Benedicto Marti C, Herrera Portal P: Indwell times, complications and costs of open vs closed safety peripheral intravenous catheters: a randomized study. Journal of Hospital Infection 2014;86:117-26.

3. Grant MS: The effect of blood drawing techniques and equipment on the hemolysis of ED laboratory blood samples. J Emerg Nurs 2003;29:116-21.

4. Ong ME, Chan YH, Lim CS: Reducing blood sample hemolysis at a tertiary hospital emergency department. Am J Med 2009;122:1054 e1-6.

5. Fry M, Jones K: The clinical initiative nurse: extending the role of the emergency nurse, who benefits? Australian Emergency Nursing Journal 2005;8:9-12.

6. Hoctor B: Nurse cannulation: introducing an advanced clinical skill. Emerg Nurse 2009;17:16-8.

7. Bolton D: Improving peripheral cannulation practice at an NHS Trust. Br J Nurs 2010;19:1346, 1348-50.

8. Goransson KE, Johansson E: Indication and usage of peripheral venous catheters inserted in adult patients during emergency care. J Vasc Access 2011;12:193-9.

9. Henderson RA, Thomson DP, Bahrs BA, Norman MP: Unnecessary intravenous access in the emergency setting. Prehosp Emerg Care 1998;2:312-6.

10. Nguyen T, Hirsh D, Khan N, Massey R, Simon H: Potential Impact of Peripheral Intravenous Catheter Placement on Resource Use in the Pediatric Emergency Department. Pediatric Emergency Care 2010;26:26-29.

11. Limm EI, Fang X, Dendle C, Stuart RL, Egerton Warburton D: Half of all peripheral intravenous lines in an Australian tertiary emergency department are unused: pain with no gain? Ann Emerg Med 2013;62:521-5.

12. Marsh N, Webster J, Mihala G, Rickard CM: Devices and dressings to secure peripheral venous catheters to prevent complications. Cochrane Database Syst Rev 2015;6:CD011070.

13. Grune F, Schrappe M, Basten J, Wenchel HM, Tual E, Stutzer H: Phlebitis rate and time kinetics of short peripheral intravenous catheters. Infection 2004;32:30-2.

14. Stuart RL, Cameron DR, Scott $C$, et al.: Peripheral intravenous catheter-associated Staphylococcus aureus bacteraemia: more than 5 years of prospective data from two tertiary health services. Med J Aust 2013;198:551-3.

15. Limm E, Fang X, Dendle C, Stuart R, Egerton Warburton D: Half of All Peripheral Intravenous Lines in an Australian Tertiary Emergency Department Are Unused: Pain With No Gain? Annals of Emergency Medicine 2013;62:521-5.

16. Fry M, Ruperto K, Jarrett K, Wheeler J, Fong J, Fetchet W: Managing the wait: clinical initiative nurses' perceptions of an extended practice role. Australasian Emergency Nursing Journal 2013;15:202- 210 . 
Table 1: Characteristics of patients with and without PIVC

1

\section{Gender}

Female

$208(58.2 \%)$

$124(59.3 \%)$

$84(56.7 \%)$

Male

$159(44.5 \%) \quad 85(40.6 \%)$

Years (Mean)

$52.9(\mathrm{SD} 23.97)$

$59(56.51)$

45 (47.97)

\section{Arrival mode}

Private transport

$224(62.7 \%)$

$130(62.2 \%)$

$94(63.5 \%)$

State Ambulance

$127(35.6 \%)$

$74(35.4 \%)$

$53(35.8 \%) \quad \mathrm{p}=.063$

Police/CS vehicle

$4(1.1 \%)$

$3(1.4 \%)$

$1(0.6 \%)$

$2(0.5 \%)$

$2(0.9 \%)$

Triage code

$(\mathbf{n}=354)$

1

$8(2.2 \%)$

$8(2.2 \%)$

2

$62(17.4 \%)$

$52(83.8 \%) \quad 10(16.0 \%)$

3

$142(39.8 \%)$

$87(61.2 \%) \quad 55(38.7 \%)$

4

$117(32.8 \%)$

$55(47.0 \%) \quad 62(53.0 \%)$

5

$25(7.0 \%)$

$5(20.0 \%)$

$20(80.0 \%)$

$3(0.8 \%)$

Missing 
ED Disposition

Discharged from ED

Admitted to Ward

Admitted to Critical care

Transferred to Hospital

Death in ED $(\mathbf{n}=357)$

$183(51.2 \%)$

$91(49.7 \%)$

$92(50.3 \%)$

$93(32.1 \%)$

$85(91.4 \%)$

$8(8.6 \%)$

$10(2.8 \%)$

$10(100 \%)$

$2(0.6 \%)$

$2(100 \%)$

11

13

14

15

16

17

18

19

20

21

22

23

24

25

26

27

28

29

30

31

32

33

34

35

36

37

38

39

40

41

42

43

44

45

46

47

48

49

50

51

52

53

54

55

56

57

58

59

60

61

62

63

64

65 
Table 2: Insertion and usage of PIVC devices

PIVC insertion $\quad \mathrm{n}=209$

\section{PIVC size}

Unknown

$157(75 \%)$

$20 \mathrm{~g}$

$30(57.6 \%)$

$18 \mathrm{~g}$

$18(34.6 \%)$

$22 \mathrm{~g}$

$4(7.6 \%)$

Insertion site

First PIVC ( $\mathrm{n}=209) \quad$ First PIVC $(\mathrm{n}=209) \quad$ Second PIVC $(\mathrm{n}=21)$

Cubital Fossa

$78(33.4 \%)$

$67(32.0 \%)$

$11(52.3 \%)$

Missing

$58(24.89 \%)$

Hand

$42(18.0 \%)$

$39(18.66 \%)$

$3(14.29 \%)$

Forearm

$29(12.4 \%) \quad 26(12.4 \%)$

$3(14.2 \%)$

Arm

$22(9.4 \%)$

$20(9.5 \%)$

$2(9.52 \%)$

Foot

$1(0.4 \%) \quad 1(0.48 \%)$

\section{PIVC Usage}

Intravenous Fluids $128(68.8 \%)$

Medications $\quad 119(63.9 \%)$

Pathology $\quad 78(41.9 \%)$

Pain management $\quad 78(41.9 \%)$ 
Contrast $\quad 9(4.8 \%)$

Other reason $\quad 5(2.6 \%)$

Deterioration $\quad 3(1.6 \%)$

\section{PIVC Interventions used per cannula}

1

$59(31.7 \%)$

2

$69(37.0 \%)$

3

$47(25.2 \%)$

4

$13(6.4 \%)$

5

$2(12.7 \%)$ 


\section{Ethical considerations}

This paper reports the findings of a study that adhered to the national statement on the conduct of human research by the Australian National Health and Medical Research and was approved by the Human Ethics Committees South East Sydney Local Health District Human Ethics Committee (13/201LNR/13/POWH/447). All participant data were immediately de-identified for the study and stored in a password protected Excel file accessible only to the investigators. 


\section{What is known}

- Insertion of peripheral cannulae can lead to inappropriate adverse patient outcomes.

- Inappropriate insertion of peripheral cannulae can result in significant costs and utilisation of staff resources.

- EDs frequently insert peripheral cannulae that are not utilized within the ED stay. inappropriately

What this paper adds

- In contrast to existing literature the insertion of peripheral cannulae was appropriate within one tertiary referral ED.

- The majority of ED peripheral cannulae were used for multiple medical interventions.

- Peripheral cannulae documentation by operator, site and attempts was poor. 